\title{
CPAP decreases plasma levels of soluble tumour necrosis factor- $\alpha$ receptor 1 in
} obstructive sleep apnoea

\author{
M.A. Arias*, F. García-Río\#, A. Alonso-Fernández", Á. Hernanz ${ }^{+}$, R. Hidalgo ${ }^{\S}$, \\ V. Martínez-Mateo*, S. Bartolomé* and L. Rodríguez-Padial*
}

ABSTRACT: There is increasing evidence that inflammation plays an important role in the development of cardiovascular complications in patients with obstructive sleep apnoea (OSA).

No previous works have studied levels of soluble tumour necrosis factor- $\alpha$ receptor (sTNFR)-1 in patients with OSA. The aims of the present study were to examine serum levels of sTNFR-1 and the effect of nasal continuous positive airway pressure (CPAP) in patients with OSA.

A prospective, randomised, placebo-controlled crossover study was performed. In total, 30 consecutive newly diagnosed OSA patients (apnoea/hypopnoea index $43.8 \pm 27.0$ events $\cdot h^{-1}$ ) and 15 healthy obese patients were selected. Urinary levels of norepinephrine and epinephrine, as well as plasma sTNFR-1, tumour necrosis factor (TNF)- $\alpha$, interleukin (IL)- 6 and leukotriene (LT) $B_{4}$ levels were obtained at baseline and after 3 months of CPAP or sham CPAP.

Nocturnal urinary levels of norepinephrine, epinephrine and sTNFR-1 (1,053 \pm 269 versus $820 \pm 166 \mathrm{pg} \cdot \mathrm{mL}^{-1}$ ) were significantly higher in OSA patients. There were no significant differences in plasma levels of IL-6, LTB no significant differences in blood pressure, urinary catecholamine levels, or plasma IL-6, LTB 4 and TNF- $\alpha$ levels after both treatment modalities. However, after 3 months of effective CPAP usage, sTNFR-1 levels were significantly reduced $\left(1,053 \pm 269\right.$ versus $\left.899 \pm 254 \mathrm{pg} \cdot \mathrm{mL}^{-1}\right)$.

Obstructive sleep apnoea patients have higher levels of soluble tumour necrosis factor- $\alpha$ receptor 1 than individuals without OSA; soluble tumour necrosis factor- $\alpha$ receptor 1 levels are lowered by continuous positive airway pressure therapy. These findings further corroborate a potential role of inflammation in the natural history of obstructive sleep apnoea.

KEYWORDS: Continuous positive airway pressure, inflammation, obstructive sleep apnoea

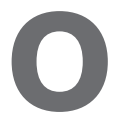

bstructive sleep apnoea (OSA) is the most common form of sleep-disordered breathing, affecting $4 \%$ of middle-aged (30-60 yrs) males and 2\% of middle-aged (3060 yrs) females. OSA is characterised by repeated partial or complete closure of the upper airway during sleep leading to chronic intermittent hypoxia, sleep fragmentation and daytime sleepiness. This medical condition is associated with a great variety of cardiovascular disturbances and stroke, resulting in severe morbidity and mortality [1]. OSA has been associated with an increased risk of systemic hypertension, left and right ventricular dysfunction, ventricular hypertrophy, heart failure, metabolic dysregulation, pulmonary hypertension, coronary artery disease and cardiac arrhythmias. There are many risk factors for OSA, including sex (male), advanced age and obesity, which are the same for cardiovascular disease, making it difficult to accurately determine the role of OSA as an independent risk factor.

Systemic inflammation is crucial in the pathogenesis of all stages of atherosclerosis. There is also increasing evidence that inflammation plays an important role in the development of cardiovascular complications in patients with OSA [2].

Conversely, several studies have demonstrated that tumour necrosis factor (TNF)- $\alpha$ and its soluble receptors, especially soluble TNF- $\alpha$ receptor (sTNFR)-1, are frequently elevated in patients with heart failure, providing useful prognostic information [3-5]. In addition, higher inflammatory marker levels may predict survival and development of overt heart failure in patients with myocardial infarction $[6,7]$ and may antedate new-onset heart failure even in patients with asymptomatic left ventricular systolic dysfunction

\section{AFFILIATIONS}

Depts of *Cardiology and

${ }^{\S}$ Pneumology, Hospital Virgen de la Salud, Toledo, and

Depts of ${ }^{\#}$ Pneumology and ${ }^{+}$Biochemistry and Molecular Biology, Hospital Universitario La Paz, Madrid, and 'Dept of Pneumology, Hospital Universitario Son Dureta, Palma de Mallorca, Spain.

CORRESPONDENCE

F. García-Río

c/Alfredo Marqueríe 11

izqda $1^{\circ} \mathrm{A}$

28034 Madrid

Spain

Fax: 34917277096

E-mail: fgr01m@gmail.com

Received:

January 152008

Accepted after revision:

May 022008

\section{SUPPORT STATEMENT}

This research was partly supported by a grant from the Fondo de Investigación Sanitaria (FIS; Spain; grant code exp. 01/0278) and Neumomadrid (Madrid, Spain; 2000).

STATEMENT OF INTEREST None declared. 
without prior myocardial infarction [8]. TNF- $\alpha$ soluble receptors have also been linked to disorders in glucose metabolism [9], and sTNFR-1 is probably an important regulator of sleep-wake cycles [10].

There are no data about the possible presence of elevated plasma sTNFR-1 levels in individuals with OSA. Moreover, previous studies evaluating the effects of continuous positive airway pressure (CPAP) therapy, the treatment of choice for OSA, on inflammatory marker levels were not examined with a randomised, placebo-controlled design [11-15]. The aims of the current study were: 1) to evaluate whether levels of sTNFR-1, as well as other pro-inflammatory cytokines, including TNF- $\alpha$, interleukin (IL)-6, and leukotriene (LT) $B_{4}$, are elevated in OSA and otherwise healthy obese patients compared with obese subjects without OSA; and 2) to determine whether treatment with CPAP decreases cytokine levels in a randomised, placebo-controlled crossover study design.

\section{MATERIALS AND METHODS Study population}

In total, 33 consecutive newly diagnosed OSA patients (apnoea/ hypopnoea index $(\mathrm{AHI}) \geqslant 10$ events $\cdot \mathrm{h}^{-1}$ and excessive daytime sleepiness (Epworth sleepiness scale $>11)$ ) and 15 obese control subjects (AHI $<5$ events $\cdot \mathrm{h}^{-1}$ and Epworth sleepiness scale $<10$ ) were selected to be studied from January 2004 to June 2005. None of the patients had ever received treatment for OSA. Control subjects were recruited from a list of healthy subjects from the present authors' Madrid metropolitan area of healthcare assigned to the Hospital La Paz (Madrid, Spain), who had undergone a routine health examination in the previous 3 months. A control subject similar in sex, age ( \pm 2 yrs), weight $( \pm 2 \mathrm{~kg})$ and height $( \pm 5 \mathrm{~cm})$ with regard to the two preceding patients was randomly selected to be included in the study. Exclusion criteria for both study groups were: 1) unwillingness or inability to perform the testing procedure; 2) obstructive or restrictive lung disease demonstrated on pulmonary function testing; 3) current use of cardioactive drugs; 4) cardiac rhythm disturbances, including sinus bradycardia and sinus tachycardia; 5) known hypertension, or 24-h mean blood pressure of 135 systolic and/or $85 \mathrm{mmHg}$ diastolic or more; 6) left ventricular ejection fraction $<50 \%$; ischaemic or valvular heart disease; hypertrophic, restrictive or infiltrative cardiomyopathy; pericardial disease or stroke by history or physical examination, ECG, chest radiography, conventional stress testing and echocardiography; 7) diabetes mellitus, by history or two random blood glucose levels $\geqslant 126 \mathrm{mg} \cdot \mathrm{dL}^{-1} ; 8$ ) morbid obesity (body mass index (BMI) $>40 \mathrm{~kg} \cdot \mathrm{m}^{-2}$ ); and 9) daytime hypoxaemia (arterial oxygen tension $<70 \mathrm{mmHg}(9.3 \mathrm{kPa})$ ) or hypercapnia (arterial carbon dioxide tension $>45 \mathrm{mmHg}(6.0 \mathrm{kPa}))$. Withdrawal criteria were: 1) clinical exacerbation leading to a change in medication; 2) hospital admission for $\geqslant 10$ days; and 3) average nightly CPAP usage $<3.5 \mathrm{~h}$. Three patients were deemed ineligible for inclusion after initial assessments (one had left ventricular systolic dysfunction secondary to ischaemic heart disease and two had unknown mitral stenosis); therefore, 30 OSA patients were finally included in the study.

The study was approved by the Institutional Ethics Committee at the Hospital Universitario La Paz (Madrid, Spain). All subjects gave their written informed consent prior to enrolment.

\section{Study protocol}

A single-centre, prospective, randomised, double-blind, placebo-controlled, crossover study was performed, in which OSA patients received effective CPAP (Aria LX CPAP device; Respironics, Murrysville, PA, USA) and sham CPAP therapy for two 12-week periods (fig. 1). The sham CPAP device consisted of a conventional CPAP device, in which the area of the exhalation port was amplified, thereby nearly cancelling nasal pressure $\left(1-2 \mathrm{cmH}_{2} \mathrm{O}(0.1-0.2 \mathrm{kPa})\right)$; an orifice resistor was connected between the tubing and the CPAP unit that loads the blower with the same airflow resistance as in effective CPAP.

OSA patients underwent a full-night CPAP titration study with an automated pressure setting device (Auto Set; ResMed, Sydney, Australia). Patients were given detailed instructions on using CPAP equipment, but they were not informed of the type of therapy they were receiving. Compliance data were measured with a run-time counter.

All subjects underwent baseline sleep study, 24-h ambulatory blood pressure monitoring (ABPM) and a transthoracic echocardiogram. Plasma levels of sTNFR-1, TNF- $\alpha$, LTB 4 and IL-6 were measured. Urine specimens were also collected. OSA patients were randomised to receive either effective or sham CPAP therapy for 12 weeks. They were then readmitted to the hospital and the CPAP device was switched to the alternative mode of therapy for another 12 weeks. Echocardiogram, ABPM, plasma cytokines and catecholamines were measured just after each period with either effective or sham CPAP treatment.

\section{Measurements}

$\mathrm{LTB}_{4}$ concentrations were measured by a forward sequential competitive enzyme immunoassay (R\&D Systems, Minneapolis, MN, USA). The inter- and intra-assay variations between the samples were 10.5 and $9.3 \%$, respectively, and the detection limit of the assay was $27.6 \mathrm{pg} \cdot \mathrm{mL}^{-1}$.

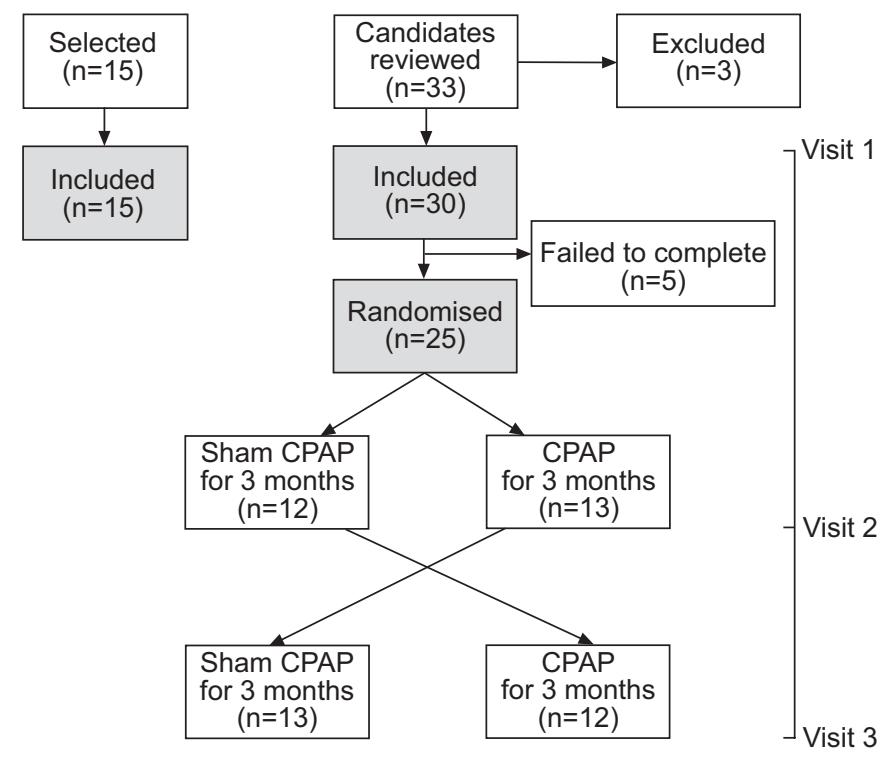

FIGURE 1. Protocol of the study. CPAP: continuous positive airway pressure. 
Quantitative sandwich enzyme immunoassay kits (R\&D Systems) were used to measure IL-6, TNF- $\alpha$ and sTNFR-1 concentrations in plasma. The inter- and intra-assay variations between the samples were as follows. IL-6: 3.3 and 1.6\%; TNF- $\alpha$ : 4.6 and $4.2 \%$; and sTNFR-1: 5.0 and $5.2 \%$, respectively, i.e. the inter- and intra-assay variabilities were $<10 \%$. The detection limits of the assays were $0.7,0.5$ and $0.43 \mathrm{pg} \cdot \mathrm{mL}^{-1}$, respectively.

During the same night that urine specimens were collected, respiratory polygraphy was performed in OSA patients and control subjects. A previously validated portable respiratory recording device was used (Sibel Home-300; Sibel S.A., Barcelona, Spain) [16], which records oronasal airflow (using a thermistor and nasal cannula prongs), chest wall impedance, oxygen saturation, snoring and body position. Oximetry data were averaged over 2-s sampling intervals. Artefacts were removed by eliminating all changes of oxygen saturation between consecutive sampling intervals of $>4 \% \cdot \mathrm{s}^{-1}$, and any oxygen saturation $<20 \%$.

Respiratory events were classified as either obstructive or central on the basis of presence or absence of respiratory effort. Respiratory events were scored as apnoea when there was a cessation of oronasal airflow lasting $\geqslant 10 \mathrm{~s}$. Hypopnoea was defined as a decrease of $50 \%$ in oronasal airflow lasting $>10 \mathrm{~s}$, associated with a fall in arterial oxygen saturation $\left(\mathrm{Sa}_{\mathrm{a}} \mathrm{O}_{2}\right)$ of $>4 \%$ of the preceding baseline level. Mean night-time $\mathrm{Sa}_{1} \mathrm{O}_{2}$, minimum $\mathrm{Sa}_{1} \mathrm{O}_{2}$ (lowest values recorded during sleep), oxygen desaturation index and percentage of time with $\mathrm{Sa}_{\mathrm{a}} \mathrm{O}_{2}<90 \%$ on nocturnal oximetry were computed as indices of nocturnal oxygen saturation.

Spirometry, echocardiogram and 24-h ABPM were performed on each patient as previously described [17, 18]. Urinary excretion of norepinephrine and epinephrine were also determined as previously described [17].

\section{Statistical analyses}

Values are expressed as mean \pm SD or percentage. All statistical tests were two-sided. The comparisons between the groups were performed using the Wilcoxon rank-sum test, while the Chisquared test was used for evaluating frequencies. Relationships between variables were determined by the Pearson's correlation analysis. Comparisons of effects of the treatments over time were made with repeated-measures ANOVA, using treatment as a within-subject factor and order as a between-subject factor. When ANOVA results showed significant differences between treatment conditions, post hoc multiple comparisons were performed with the Bonferroni test. A value of $\mathrm{p}<0.05$ was considered statistically significant.

\section{RESULTS}

All individuals in both groups were male. Characteristics of the studied population are shown in table 1 . There were no differences in either demographic, blood pressure or lung function data between the two study groups. Nocturnal levels of norepinephrine and epinephrine were higher in patients with OSA. Nocturnal epinephrine dip was higher in control subjects. Although the values were within the normal limits, OSA patients had higher values of left ventricular posterior
TABLE 1 Baseline characteristics in obstructive sleep apnoea (OSA) patients and control subjects

\begin{tabular}{|c|c|c|c|}
\hline & $\begin{array}{l}\text { OSA } \\
\text { group }\end{array}$ & $\begin{array}{l}\text { Control } \\
\text { group }\end{array}$ & $p$-value ${ }^{\#}$ \\
\hline Subjects n & 30 & 15 & \\
\hline Age yrs & $52 \pm 13$ & $48 \pm 10$ & 0.292 \\
\hline Height $\mathrm{cm}$ & $171 \pm 7$ & $173 \pm 5$ & 0.264 \\
\hline Weight kg & $89 \pm 14$ & $86 \pm 16$ & 0.546 \\
\hline$B M I \mathbf{~ k g} \cdot \mathbf{m}^{-2}$ & $30.5 \pm 4.0$ & $28.7 \pm 4.7$ & 0.188 \\
\hline Current smokers \% & 37 & 27 & 0.374 \\
\hline Smoking history pack-yrs & $20 \pm 12$ & $20 \pm 7$ & 0.965 \\
\hline AHI events $\cdot h^{-1}$ & $43.8 \pm 27.0$ & $3.7 \pm 3.3$ & 0.000 \\
\hline Al events $\cdot h^{-1}$ & $29.4 \pm 26.4$ & $1.0 \pm 0.8$ & 0.005 \\
\hline Obstructive events \% & $96 \pm 6$ & $66 \pm 31$ & 0.000 \\
\hline $\begin{array}{l}\text { Total sleep study time } \\
\quad<\mathbf{9 0} \% \mathrm{Sa}_{\mathrm{a}} \mathrm{O}_{2} \%\end{array}$ & $18.41 \pm 23.39$ & $0.04 \pm 0.05$ & 0.000 \\
\hline ODI events $\cdot h^{-1}$ & $44.6 \pm 26.7$ & $4.7 \pm 3.8$ & 0.000 \\
\hline Mean nocturnal $\mathrm{Sa}, \mathrm{O}_{2} \%$ & $91 \pm 6$ & $94 \pm 2$ & 0.024 \\
\hline Minimum $\mathrm{Sa}, \mathrm{O}_{2} \%$ & $72 \pm 15$ & $85 \pm 5$ & 0.016 \\
\hline FVC \% pred & $105 \pm 17$ & $99 \pm 16$ & 0.313 \\
\hline FEV $1 \%$ pred & $111 \pm 15$ & $108 \pm 16$ & 0.500 \\
\hline FEV $1 /$ FVC $\%$ & $85.8 \pm 7.2$ & $88.2 \pm 3.6$ & 0.156 \\
\hline Daytime SBP mmHg & $126 \pm 10$ & $122 \pm 9$ & 0.154 \\
\hline Daytime DBP mmHg & $79 \pm 6$ & $78 \pm 5$ & 0.529 \\
\hline Daytime MBP mmHg & $95 \pm 7$ & $92 \pm 6$ & 0.208 \\
\hline Night-time SBP mmHg & $117 \pm 11$ & $110 \pm 10$ & 0.059 \\
\hline Night-time DBP mmHg & $70 \pm 7$ & $67 \pm 6$ & 0.127 \\
\hline Night-time MBP mmHg & $86 \pm 8$ & $82 \pm 8$ & 0.058 \\
\hline Diurnal norepine $\mu \mathrm{g} \cdot \mathbf{g}^{-1}$ & $34.9 \pm 13.1$ & $29.9 \pm 11.6$ & 0.211 \\
\hline Diurnal epine $\mu \mathrm{g} \cdot \mathrm{g}^{-1}$ & $7.0 \pm 4.9$ & $7.9 \pm 4.5$ & 0.571 \\
\hline Nocturnal norepine $\mu \mathrm{g} \cdot \mathbf{g}^{-1}$ & $21.9 \pm 12.1$ & $12.9 \pm 6.2$ & 0.002 \\
\hline Nocturnal epine $\mu \mathrm{g} \cdot \mathrm{g}^{-1}$ & $6.5 \pm 5.3$ & $4.2 \pm 2.4$ & 0.049 \\
\hline Norepine nocturnal dip \% & $36 \pm 27$ & $48 \pm 24$ & 0.182 \\
\hline Epine nocturnal dip \% & $16 \pm 10$ & $32 \pm 46$ & 0.039 \\
\hline Heart rate beats $\cdot \mathrm{min}^{-1}$ & $76 \pm 13$ & $78 \pm 11$ & 0.640 \\
\hline LVDD mm & $52.1 \pm 2.8$ & $50.7 \pm 5.8$ & 0.399 \\
\hline LVSD mm & $32.5 \pm 2.5$ & $30.3 \pm 4.8$ & 0.060 \\
\hline Posterior wall mm & $10.6 \pm 1.2$ & $9.3 \pm 1.4$ & 0.004 \\
\hline Interventricular septum mm & $10.7 \pm 1.1$ & $9.5 \pm 1.5$ & 0.009 \\
\hline Left ventricular mass $\mathbf{g}$ & $213.4 \pm 38.0$ & $178.3 \pm 39.5$ & 0.007 \\
\hline $\begin{array}{l}\text { Left ventricular mass } \\
\text { index } \mathbf{g} \cdot \mathrm{m}^{-2}\end{array}$ & $107.0 \pm 19.6$ & $89.5 \pm 17.6$ & 0.007 \\
\hline LVSF \% & $37.6 \pm 3.1$ & $40.1 \pm 4.8$ & 0.076 \\
\hline LVEF \% & $67.1 \pm 3.7$ & $70.1 \pm 5.9$ & 0.084 \\
\hline Left atrial diameter mm & $37.6 \pm 3.6$ & $36.1 \pm 5.6$ & 0.363 \\
\hline
\end{tabular}

Data are expressed as mean $\pm S D$, unless otherwise stated. BMI: body mass index; $\mathrm{AHI}$ : apnoea/hypopnoea index; Al: apnoea index; $\mathrm{Sa}_{2} \mathrm{O}_{2}$ : arterial oxygen saturation; ODI: oxygen desaturation index; FVC: forced vital capacity; \% pred: $\%$ predicted; FEV1: forced expiratory volume in one second; SBP: systolic blood pressure; DBP: diastolic blood pressure; MBP: mean blood pressure; norepine: norepinephrine; epine: epinephrine; LVDD: left ventricular diastolic diameter; LVSD: left ventricular systolic diameter; LVSF: left ventricular shortening fraction; LVEF: left ventricular ejection fraction. ${ }^{\#}$ : Wilcoxon test. 


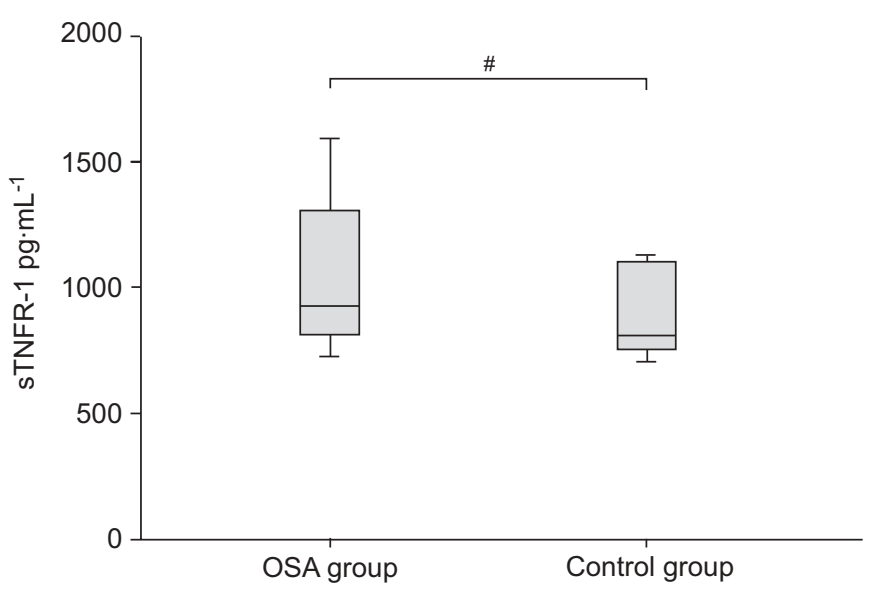

FIGURE 2. Serum soluble tumour necrosis factor- $\alpha$ receptor (sTNFR)-1 levels in obstructive sleep apnoea (OSA) patients and control subjects. Box and whisker plots represent median, interquartile range and range. ${ }^{*}: p=0.032$ by Wilcoxon test.

wall, interventricular septum thickness, left ventricular mass, and left ventricular mass index.

Baseline levels of sTNFR-1 were higher in OSA patients compared with control obese subjects (fig. 2). There was no significant difference in TNF- $\alpha$, IL-6 or $\mathrm{LTB}_{4}$ levels between the two study groups (table 2).

Several associations between baseline variables and cytokine levels were revealed. There were no significant relationships between plasma levels of TNF- $\alpha$ or $\mathrm{LTB}_{4}$ and any studied variable. Significant positive relationships were found between weight and sTNFR-1 $(\mathrm{r}=0.446, \mathrm{p}=0.033)$ and IL-6 $(\mathrm{r}=0.736$, $\mathrm{p}=0.003$ ) levels. Also, BMI was associated with high sTNRF-1 $(r=0.414, p=0.049)$ and IL-6 $(r=0.693, p=0.006)$ levels. Low epinephrine nocturnal dip was associated with higher sTNFR-1 ( $\mathrm{r}=-0.484, \mathrm{p}=0.019)$ levels. In addition, levels of IL-6 were positively correlated with diurnal norepinephrine levels $(r=0.667, p=0.009)$ and negatively correlated with forced vital capacity $\%$ predicted $(r=-0.636, p=0.015)$ and forced expiratory volume in one second $\%$ pred $(r=-0.583, p=0.029)$.

Pearson's correlation coefficients between levels of sTNFR-1, TNF- $\alpha$, IL- 6 and $\mathrm{LTB}_{4}$ and sleep parameters in patients with OSA are shown in table 3 . There was no significant relationship

\begin{tabular}{|c|c|c|c|}
\hline \multirow[t]{2}{*}{ TABLE 2} & \multicolumn{3}{|c|}{$\begin{array}{l}\text { Plasma levels of pro-inflammatory cytokines in } \\
\text { obstructive sleep apnoea (OSA) patients and } \\
\text { control subjects }\end{array}$} \\
\hline & OSA group ${ }^{\#}$ & Control group & p-value ${ }^{+}$ \\
\hline IL-6 pg $\cdot \mathrm{mL}^{-1}$ & $4.2 \pm 3.4$ & $3.6 \pm 1.8$ & 0.523 \\
\hline $\mathrm{LTB}_{4} \mathbf{n g} \cdot \mathrm{mL}^{-1}$ & $5972 \pm 8239$ & $7650 \pm 18033$ & 0.780 \\
\hline TNF- $\alpha \mathrm{pg} \cdot \mathrm{mL}^{-1}$ & $18.5 \pm 13.4$ & $11.4 \pm 12.2$ & 0.526 \\
\hline sTNFR-1 pg.mL & $1053 \pm 269$ & $820 \pm 166$ & 0.032 \\
\hline \multicolumn{4}{|c|}{$\begin{array}{l}\text { Data are expressed as mean } \pm \mathrm{SD} \text {, unless otherwise stated. IL: interleukin; LT: } \\
\text { leukotriene; TNF: tumour necrosis factor; sTNFR: soluble TNF- } \alpha \text { receptor. }{ }^{\#} \text { : } \\
n=30 ;{ }^{\bullet}: \mathrm{n}=15{ }^{+}{ }^{\text {: Wilcoxon test. }}\end{array}$} \\
\hline
\end{tabular}

between any of the sleep parameters and circulating cytokine levels.

Five OSA patients failed to complete the study, two because of an average nightly usage of CPAP of $<3.5 \mathrm{~h}$, and three were lost from follow-up. Complete measurements were available in 25 patients. Mean CPAP pressure was $10 \pm 2 \mathrm{cmH}_{2} \mathrm{O}(0.98 \pm 0.20 \mathrm{kPa})$, and the average nightly usage was similar on sham CPAP and effective CPAP $(6.3 \pm 1.6$ versus $6.2 \pm 1.1 \mathrm{~h} ; \mathrm{p}=0.6636)$. There were no differences in heart rate, weight, blood pressure recordings or urinary catecholamines at baseline and after sham or effective CPAP in OSA patients (table 4). There was no randomisation of patients in the cross-over design. After 12 weeks on effective CPAP, a significant reduction in sTNFR-1 levels was observed (table 5 and fig. 3). After both modes of treatment, no significant differences were observed in either systolic function or ventricular structure parameters.

\section{DISCUSSION}

In the present study, levels of sTNFR-1 were significantly higher in obese OSA and otherwise healthy patients compared with obese control subjects. In addition, circulating TNF- $\alpha$, IL-6 and $\mathrm{LTB}_{4}$ levels were not different between the two study groups. Interestingly, after 3 months of CPAP treatment, circulating sTNFR-1 levels were lower in OSA patients.

It has been shown that elevated sTNFR-1 levels predict the incidence of fatal and nonfatal cardiovascular events in several conditions, such as heart failure and acute coronary syndrome [3-7]. In that sense, in patients with acute myocardial infarction sTNFR-1 levels have been shown to be a major predictor of mortality and heart failure [6, 7]. Increasing circulating levels of sTNFR-1 are also associated with increased mortality in a wide range of heart failure patients [3-5]. Reported levels of sTNFR-1 in both heart failure and ischaemic patients were higher than those observed in the present OSA patient population, probably reflecting the fact that the present

\begin{tabular}{|c|c|c|c|c|c|}
\hline \multirow[t]{2}{*}{ TABLE 3} & \multicolumn{5}{|c|}{$\begin{array}{l}\text { Correlation between plasma cytokine levels and } \\
\text { sleep parameters in obstructive sleep apnoea } \\
\text { patients }\end{array}$} \\
\hline & & IL-6 & $\mathrm{LTB}_{4}$ & TNF & sTNFR-1 \\
\hline \multicolumn{6}{|l|}{ AHI } \\
\hline \multicolumn{2}{|c|}{ Correlation coefficient $r$} & 0.237 & -0.203 & 0.123 & 0.104 \\
\hline \multicolumn{2}{|c|}{$p$-values } & 0.414 & 0.506 & 0.478 & 0.638 \\
\hline \multicolumn{6}{|l|}{ ODI } \\
\hline \multicolumn{2}{|c|}{ Correlation coefficient $r$} & 0.396 & -0.101 & 0.241 & 0.321 \\
\hline \multicolumn{2}{|c|}{$\mathrm{p}$-values } & 0.203 & 0.754 & 0.124 & 0.156 \\
\hline \multicolumn{6}{|c|}{ Mean nocturnal $\mathrm{Sa}, \mathrm{O}_{2}$} \\
\hline \multicolumn{2}{|c|}{ Correlation coefficient $r$} & -0.491 & 0.211 & 0.024 & 0.014 \\
\hline \multicolumn{2}{|c|}{$p$-values } & 0.075 & 0.489 & 0.781 & 0.811 \\
\hline \multicolumn{6}{|c|}{ Minimum $\mathrm{Sa}, \mathrm{O}_{2}$} \\
\hline \multicolumn{2}{|c|}{ Correlation coefficient $r$} & -0.209 & 0.140 & 0.011 & 0.053 \\
\hline \multicolumn{2}{|c|}{ p-values } & 0.473 & 0.649 & 0.825 & 0.811 \\
\hline
\end{tabular}

IL: interleukin; LT: leukotriene; TNF: tumour necrosis factor; sTNFR: soluble tumour necrosis factor- $\alpha$ receptor; AHI: apnoea/hypopnoea index; ODI: oxygen desaturation index; $\mathrm{Sa}, \mathrm{O}_{2}$ : arterial oxygen saturation 


\begin{tabular}{|c|c|c|c|c|}
\hline \multirow[t]{2}{*}{ TABLE 4} & \multicolumn{4}{|c|}{$\begin{array}{l}\text { Weight, blood pressure data, and urinary } \\
\text { catecholamines in } 25 \text { obstructive sleep apnoea } \\
\text { patients at baseline and after both sham } \\
\text { continuous positive airway pressure (CPAP) and } \\
\text { effective CPAP }\end{array}$} \\
\hline & & Baseline & Sham CPAP & CPAP \\
\hline \multicolumn{2}{|l|}{ Weight kg } & $89 \pm 14$ & $89 \pm 15$ & $89 \pm 16$ \\
\hline \multicolumn{2}{|c|}{$\mathrm{BMI} \mathbf{k g} \cdot \mathrm{m}^{-2}$} & $30.4 \pm 4.0$ & $30.3 \pm 4.1$ & $30.2 \pm 4.4$ \\
\hline \multicolumn{2}{|c|}{ Heart rate beats $\cdot \min ^{-1}$} & $76 \pm 13$ & $72 \pm 11$ & $79 \pm 20$ \\
\hline \multicolumn{2}{|c|}{ Daytime SBP mmHg } & $126 \pm 10$ & $126 \pm 12$ & $127 \pm 9$ \\
\hline \multicolumn{2}{|c|}{ Daytime DBP mmHg } & $79 \pm 6$ & $77 \pm 6$ & $78 \pm 5$ \\
\hline \multicolumn{2}{|c|}{ Daytime MBP mmHg } & $95 \pm 7$ & $94 \pm 8$ & $95 \pm 7$ \\
\hline \multicolumn{2}{|c|}{ Night-time SBP mmHg } & $118 \pm 12$ & $117 \pm 12$ & $117 \pm 17$ \\
\hline \multicolumn{2}{|c|}{ Night-time DBP mmHg } & $70 \pm 8$ & $70 \pm 6$ & $69 \pm 9$ \\
\hline \multicolumn{2}{|c|}{ Night-time MBP mmHg } & $86 \pm 9$ & $86 \pm 7$ & $86 \pm 12$ \\
\hline \multicolumn{2}{|c|}{ Diurnal norepine $\mu \mathrm{g} \cdot \mathrm{g}^{-1}$} & $36.7 \pm 13.8$ & $34.2 \pm 13.1$ & $31.3 \pm 12.0$ \\
\hline \multicolumn{2}{|c|}{ Diurnal epine $\mu \mathrm{g} \cdot \mathrm{g}^{-1}$} & $7.4 \pm 5.8$ & $7.7 \pm 3.4$ & $7.8 \pm 4.4$ \\
\hline \multicolumn{2}{|c|}{ Nocturnal norepine $\mu \mathrm{g} \cdot \mathbf{g}^{-1}$} & $23.7 \pm 12.8$ & $22.6 \pm 12.7$ & $20.1 \pm 10.0$ \\
\hline \multicolumn{2}{|c|}{ Nocturnal epine $\mu \mathrm{g} \cdot \mathrm{g}^{-1}$} & $6.8 \pm 5.9$ & $6.1 \pm 5.3$ & $6.3 \pm 4.3$ \\
\hline
\end{tabular}

Data are presented as mean $\pm \mathrm{SD}$. BMI: body mass index; SBP: systolic blood pressure; DBP: diastolic blood pressure; MBP: mean blood pressure; norepine: norepinephrine; epine: epinephrine. There were no significant differences at any time point (repeated-measures ANOVA). There was not a randomisation of patients in the crossover design.

OSA patients were free of any overt cardiovascular disease. Whether higher levels of sTNFR-1 are present in a nonselected population of OSA subjects needs to be determined in future studies. Heart failure and coronary artery disease share multiple cardiovascular pathophysiological mechanisms with OSA, including endothelial dysfunction, systemic inflammation, impaired baroreflex and sympathetic overactivity.

Elevated sTNFR-1 levels are also reported to be associated with type-2 diabetes mellitus, regardless of obesity [9]. It is conceivable that the association of OSA and high sTNFR-1 levels may raise the rate of cardiovascular events in this patient population. Increased plasma levels of pro-inflammatory cytokines may be linked not only to poor cardiovascular outcomes in OSA but also to excessive daytime sleepiness, which is a typical feature of OSA [19]. Moreover, elevated circulating cytokine levels may be the result of the enhanced cellular release of such cytokines in response to intermittent apnoea-related hypoxia, supporting a direct role for inflammation as a potential mechanism of atherogenesis in OSA [2, 20, 21]. In this regard, OSA and chronic intermittent hypoxaemia are associated with the activation of nuclear factor $\kappa B$, which is involved in the transcription of numerous genes involved in the inflammatory cascade; its downstream products (including the TNF- $\alpha$ system) may be key factors in the link between OSA and cardiovascular diseases. However, further studies are needed in order to evaluate whether increased sTNFR-1 levels can play a role in predicting subsequent prognosis during long-term follow-up in the entire spectrum of individuals with OSA due to initial inflammatory responses, or whether, on the contrary, they are an epiphenomenon secondary to sleep deprivation.

\begin{tabular}{|c|c|c|c|}
\hline \multirow[t]{2}{*}{ TABLE 5} & \multicolumn{3}{|c|}{$\begin{array}{l}\text { Plasma levels of pro-inflammatory cytokines in } \\
25 \text { obstructive sleep apnoea patients at baseline } \\
\text { and after both sham continuous positive airway } \\
\text { pressure (CPAP) and effective CPAP }\end{array}$} \\
\hline & Baseline & Sham CPAP & CPAP \\
\hline IL-6 pg $\cdot \mathrm{mL}^{-1}$ & $4.3 \pm 3.5$ & $4.6 \pm 2.4$ & $4.7 \pm 2.0$ \\
\hline $\mathrm{LTB}_{4} \mathrm{ng} \cdot \mathrm{mL}^{-1}$ & $4961 \pm 7433$ & $4456 \pm 11818$ & $4583 \pm 1243$ \\
\hline $\mathrm{TNF}-\alpha \mathrm{pg} \cdot \mathrm{mL}^{-1}$ & $18.2 \pm 12.6$ & $17.9 \pm 11.4$ & $15.7 \pm 10.9$ \\
\hline $\mathrm{sTNFR}-1 \mathrm{pg} \cdot \mathrm{mL}^{-}$ & $1103 \pm 269$ & $1132 \pm 347$ & $899 \pm 254^{\#}$ \\
\hline
\end{tabular}

Data are expressed as mean \pm SD. IL: interleukin; LT: leukotriene; TNF: tumour necrosis factor; sTNFR: soluble TNF- $\alpha$ receptor. ${ }^{\#}: p<0.05$ versus baseline and $p<0.05$ versus sham CPAP (repeated-measures ANOVA)

Previous studies have reported that patients with OSA develop systemic inflammation with increased levels of circulating proinflammatory cytokines, such as TNF- $\alpha$, IL- 6 and others, as compared with healthy subjects, and CPAP therapy reduces the levels of OSA-induced inflammatory cytokines [11-13, 15, 22]. Importantly, the increased levels of cytokines correlated with OSA severity, regardless of obesity [23]. Conversely, some authors have reported no increased levels of inflammatory mediators in individuals with OSA when compared with subjects without OSA [14, 24, 25]. In the present study, only sTNFR-1 levels were higher in OSA patients than in control subjects, but no differences were found for the other proinflammatory markers studied. These opposing results may be due to differences in the duration of the disease in the study samples, variable presence of comorbid conditions or the measurement techniques used. Cellular actions of TNF- $\alpha$ are mediated by TNF receptors. Circulating levels of TNF- $\alpha$ are frequently undetectable or highly variable in humans and sTNFR-1 levels may be a better marker for the biological effects and activation status of the TNF- $\alpha$ system.

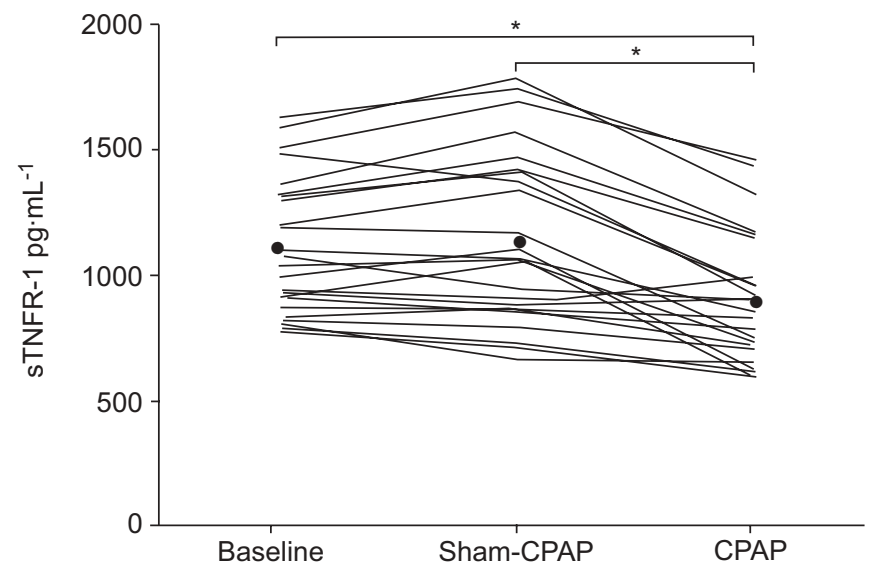

FIGURE 3. Individual serum soluble tumour necrosis factor- $\alpha$ receptor (sTNFR)-1 levels at baseline, after sham continuous positive airway pressure (CPAP) and CPAP therapy in 25 obstructive sleep aponea patients. ${ }^{*}: p<0.05$. mean values. 
In the present study, two groups of obese individuals were studied. A positive correlation was found between sTNFR-1, IL-6 levels and BMI in OSA patients, but there were no significant relationships between sleep parameters and plasma cytokine levels. However, BMI did not differ between the two study groups. Obesity is very common among OSA patients and it may also be associated with inflammation. In view of the current results, the present authors speculate that at the initial stages of the disease, when clinical cardiovascular disturbances have not yet developed, the enhanced sympathetic activity secondary to repetitive nocturnal obstructive respiratory events may be the predominant mechanism responsible for the rise in sTNFR-1 levels. An ongoing prospective randomised clinical trial is studying whether the increased risk of cardiovascular complications in individuals with OSA is caused primarily by the inflammatory effects of OSA or obesity [26].

The present study has several limitations. All individuals were males. Therefore, the findings may not be readily extrapolated to female patients. In addition, the present study included a limited number of patients. That is explained by the fact that the process of including only newly diagnosed middle-aged OSA and otherwise healthy patients to achieve the purpose of the present study was very difficult. As is well known, in the global population of patients with OSA the presence of coexistent comorbid conditions is very common. The present data do not allow an analysis of whether the arousal index is related to TNF- $\alpha$ and STNFR-1, since the patients were diagnosed by means of polygraphy. Since the principal target of the present study was to value the effect of the CPAP on serum levels of sTNFR-1 in OSA patients, neither the design nor the sample size are suitable for the evaluation of the possible determinants of the levels of sTNFR-1 in these patients. However, the present authors speculate that the impact of sleep fragmentation on sTNFR-1 levels in OSA patients might be considered to be discrete. Total sleep deprivation increases the plasma levels of sTNFR-1 and IL-6, but there have not been any identified changes associated with a partial sleep deprivation, which corresponds better to the situation of the OSA patients. Finally, the present study did not use polysomnography, but a portable respiratory recording device, to diagnose OSA. Although conventional polysomnography is considered the gold standard for the diagnosis of OSA, it has been previously demonstrated that an accurate diagnosis of OSA can be made without using neurological variables, even in an unattended setting, suggesting that the portable respiratory recording device is therefore suitable for use in epidemiological studies [27].

In conclusion, high circulating soluble tumour necrosis factor- $\alpha$ receptor-1 levels may be observed in obese obstructive sleep apnoea patients with no associated comorbid conditions. Further studies will determine whether it could serve as an early marker of ongoing systemic inflammation in obstructive sleep apnoea, increasing the risk for eventual future cardiovascular adverse events. Chronic continuous positive airway pressure application might be useful in reducing the potential cardiovascular risks of elevated circulating plasma soluble tumour necrosis factor- $\alpha$ receptor- 1 and other pro-inflammatory cytokines in patients with obstructive sleep apnoea.

\section{REFERENCES}

1 Marin JM, Carrizo SJ, Vicente E, Agusti AG. Long-term cardiovascular outcomes in men with obstructive sleep apnoea-hypopnoea with or without treatment with continuous positive airway pressure: an observational study. Lancet 2005; 365: 1046-1053.

2 Ryan S, Taylor CT, McNicholas WT. Selective activation of inflammatory pathways by intermittent hypoxia in obstructive sleep apnea syndrome. Circulation 2005; 112: 2660-2667.

3 Deswal A, Petersen NJ, Feldman AM, Young JB, White BG, Mann DL. Cytokines and cytokine receptors in advanced heart failure: an analysis of the cytokine database from the Vesnarinone trial (VEST). Circulation 2001; 103: 2055-2059.

4 Ferrari R, Bachetti T, Confortini R, et al. Tumor necrosis factor soluble receptors in patients with various degrees of congestive heart failure. Circulation 1995; 92: 1479-1486.

5 Rauchhaus M, Doehner W, Francis DP, et al. Plasma cytokine parameters and mortality in patients with chronic heart failure. Circulation 2000; 102: 3060-3067.

6 Ueland T, Kjekshus J, Froland SS, et al. Plasma levels of soluble tumor necrosis factor receptor type I during the acute phase following complicated myocardial infarction predicts survival in high-risk patients. J Am Coll Cardiol 2005; 46: 2018-2021.

7 Valgimigli M, Ceconi C, Malagutti P, et al. Tumor necrosis factor $\alpha$ receptor 1 is a major predictor of mortality and new-onset heart failure in patients with acute myocardial infarction: the Cytokine-Activation and Long-Term Prognosis in Myocardial Infarction (C-ALPHA) study. Circulation 2005; 111: 863-870.

8 Vasan RS, Sullivan LM, Roubenoff R, et al. Inflammatory markers and risk of heart failure in elderly subjects without prior myocardial infarction: the Framingham Heart Study. Circulation 2003; 107: 1486-1491.

9 Bullo M, Garcia-Lorda P, Salas-Salvado J. Plasma soluble tumor necrosis factor- $\alpha$ receptors and leptin levels in normal-weight and obese women: effect of adiposity and diabetes. Eur J Endocrinol 2002; 146: 325-331.

10 Fang J, Wang Y, Krueger JM. Mice lacking the TNF $55 \mathrm{kDa}$ receptor fail to sleep more after TNF- $\alpha$ treatment. J Neurosci 1997; 17: 5949-5955.

11 Minoguchi K, Tazaki T, Yokoe T, et al. Elevated production of tumor necrosis factor- $\alpha$ by monocytes in patients with obstructive sleep apnea syndrome. Chest 2004; 126: 1473-1479.

12 Ohga E, Tomita T, Wada H, Yamamoto $H$, Nagase $T$, Ouchi Y. Effects of obstructive sleep apnea on circulating ICAM-1, IL-8, and MCP-1. J Appl Physiol 2003; 94: 179-184.

13 Ryan S, Taylor CT, McNicholas WT. Predictors of elevated nuclear factor- $\kappa B-d e p e n d e n t$ genes in obstructive sleep apnea syndrome. Am J Respir Crit Care Med 2006; 174: 824-830.

14 Ryan S, Nolan G, Hannigan E, Cunningham S, Taylor CT, McNicholas WT. Cardiovascular risk markers in obstructive sleep apnoea syndrome and correlation with obesity. Thorax 2007; 62: 509-514.

15 Yokoe T, Minoguchi K, Matsuo H, et al. Elevated levels of C-reactive protein and interleukin-6 in patients with obstructive sleep apnea syndrome are decreased by nasal continuous positive airway pressure. Circulation 2003; 107: 1129-1134. 
16 Ballester E, Solans M, Vila X, et al. Evaluation of a portable respiratory recording device for detecting apnoeas and hypopnoeas in subjects from a general population. Eur Respir J 2000; 16: 123-127.

17 Arias MA, Garcia-Rio F, Alonso-Fernandez A, Mediano O, Martinez I, Villamor J. Obstructive sleep apnea syndrome affects left ventricular diastolic function: effects of nasal continuous positive airway pressure in men. Circulation 2005; 112: 375-383.

18 Garcia-Rio F, Pino JM, Alonso A, et al. White coat hypertension in patients with obstructive sleep apneahypopnea syndrome. Chest 2004; 125: 817-822.

19 Krueger JM, Obal FJ, Fang J, Kubota T, Taishi P. The role of cytokines in physiological sleep regulation. Ann N Y Acad Sci 2001; 933: 211-221.

20 Minoguchi K, Yokoe T, Tazaki T, et al. Increased carotid intima-media thickness and serum inflammatory markers in obstructive sleep apnea. Am J Respir Crit Care Med 2005; 172: 625-630.

21 Savransky V, Nanayakkara A, Li J, et al. Chronic intermittent hypoxia induces atherosclerosis. Am J Respir Crit Care Med 2007; 175: 1290-1297.
22 Shamsuzzaman AS, Winnicki M, Lanfranchi $\mathrm{P}$, et al. Elevated C-reactive protein in patients with obstructive sleep apnea. Circulation 2002; 105: 2462-2464.

23 Ciftci TU, Kokturk O, Bukan N, Bilgihan A. The relationship between serum cytokine levels with obesity and obstructive sleep apnea syndrome. Cytokine 2004; 28: 87-91.

24 Guilleminault C, Kirisoglu C, Ohayon MM. C-reactive protein and sleep-disordered breathing. Sleep 2004; 27: 1507-1511.

25 Imagawa S, Yamaguchi Y, Ogawa K, et al. Interleukin-6 and tumor necrosis factor- $\alpha$ in patients with obstructive sleep apnea-hypopnea syndrome. Respiration 2004; 71: 24-29.

26 Samaha FF, Giesler JC. Inflammatory response to sleep apnea in obese subjects: the Cardiovascular Effects of Obstructive Sleep Apnea (COSA) Study. www.clinicaltrials.gov Identifier NCT00371293.

27 Collop NA, Anderson WM, Boehlecke B, et al. Clinical guidelines for the use of unattended portable monitors in the diagnosis of obstructive sleep apnea in adult patients. Portable Monitoring Task Force of the American Academy of Sleep Medicine. J Clin Sleep Med 2007; 3: 737-747. 